ESAIM: M2AN 49 (2015) 1285-1302

DOI: $10.1051 / \mathrm{m} 2 \mathrm{an} / 2015012$
ESAIM: Mathematical Modelling and Numerical Analysis

www.esaim-m2an.org

\title{
COMPUTING QUANTITIES OF INTEREST FOR RANDOM DOMAINS WITH SECOND ORDER SHAPE SENSITIVITY ANALYSIS
}

\author{
Marc Dambrine ${ }^{1}$, Helmut Harbrecht ${ }^{2}$ and Bénédicte Puig ${ }^{1}$
}

\begin{abstract}
We consider random perturbations of a given domain. The characteristic amplitude of these perturbations is assumed to be small. We are interested in quantities of interest which depend on the random domain through a boundary value problem. We derive asymptotic expansions of the first moments of the distribution of this output function. A simple and efficient method is proposed to compute the coefficients of these expansions provided that the random perturbation admits a lowrank spectral representation. By numerical experiments, we compare our expansions with Monte-Carlo simulations.
\end{abstract}

Mathematics Subject Classification. 60G35, 65N75, 65N99.

Received July 18, 2014. Revised February 9, 2015.

Published online August 18, 2015.

\section{INTRODUCTION}

Many practical problems in engineering lead to boundary value problems for an unknown function which needs to be computed to obtain a real quantity of interest. In structural mechanics, the equations of elasticity are usually considered and solved to compute the leading mode of a structure or its compliance. Usually, the input parameters of the model like the geometry or the physical coefficients (typically the value of the Young modulus or Poisson ratio) are not perfectly known. It is therefore important to take these uncertainties into account.

In this work, we consider uncertainties in the geometric definition of the domain. Such uncertainties are motivated by tolerances in the fabrication process or by a damaged boundary during the life of a mechanical device. Manufactured devices are close to a nominal geometry but differ of course from its mathematical definition. Since we are motivated by tolerances, we can make the crucial assumption of the smallness of the random perturbations. This means that we assume that the non-dimensional ratio $\varepsilon$ between the characteristic size of the perturbation and the characteristic size of the domain is small. By identifying domains with their boundary, domains close to the nominal domain $D$ can be seen as a normal perturbation of the nominal boundary $\partial D$. In that case, the random domain $D(\omega)$ can be defined thanks to a real valued random field $X$ over $\partial D$ according to

$$
\partial D(\omega)=\{x+X(x, \omega) \boldsymbol{n}(x) ; x \in \partial D\},
$$

Keywords and phrases. Random domain, second order shape sensitivity, low-rank approximation.

1 Université de Pau et des Pays de l'Adour, 64000 Pau, France. marc.dambrine@univ-pau.fr; bpuig@univ-pau.fr

2 Universität Basel. helmut.harbrecht@unibas.ch 
where $\boldsymbol{n}(x)$ denotes the outer unit normal field at a point $x$ in $\partial D$. In order to take into account the uncertain geometrical definition in the numerical simulation, we have to incorporate the randomness of the computational domain into the underlying model equations. As a consequence, the quantities of engineering interest are also random. We address thus the following problem: given a complete probabilistic description of the random perturbation of the nominal boundary, compute as much information as possible to the distribution of the quantity of interest.

The most common approach to study boundary value problems with stochastic inputs is the Monte-Carlo method. In many situations, this approach is easy to implement since it requires only a large number of samples. However, for boundary values problems on random domains, each sample means a new domain and thus a new mesh, the building of new mass and stiffness matrices, etc. All these steps are mandatory to compute the quantity of interest. Therefore, the Monte-Carlo method is extremely costly and not so easy to implement in our context. This work is a contribution to the development of cheap and deterministic numerical methods to recover statistical information on the distribution of the output quantity of interest. The main advantage of the approach we propose here is to use a single mesh and to build once for all the matrices.

The smallness assumption formalized in the sequel allows us to use a sensitivity analysis with respect to geometrical perturbations of the boundary. We apply shape calculus to perform a second order sensitivity analysis and compute asymptotic expansions of the moments of the output quantity's distribution with respect to the smallness parameter $\varepsilon$ of the random perturbation. In particular, we obtain in this work a third order expansion for the expectation and a fourth order expansion for the variance. It turns out that the leading coefficients of these asymptotic expansions do only depend on the autocovariance of the random field which defines the random perturbation. We therefore use the associated integral operator to compute a low-rank approximation of the random field of the type

$$
X(x, \omega)=\sum_{i=1}^{N} \alpha_{i}(\omega) f_{i}(x) .
$$

For such a random field, we derive an analytic expression of the coefficients of the previous asymptotic expansions that are very cheap to compute: only $N+1$ boundary values problems are to be solved on the nominal geometry $D$ in the examples we present in this work. Note that $2 N+2$ problems are needed when the derivatives are evaluated thanks to an adjoint state.

We mention that random shape functionals have also been considered in [15] by means of a first order perturbation analysis. A rather general framework of the first order perturbation analysis for functionals with random input parameters, particularly random domains, has been presented [5]. To obtain more precise approximations, the present paper is based on a second order perturbation analysis of the random shape functional under consideration. Thus, the shape Hessian will enter the asymptotic expansions which makes the computations much harder. However, the shape Hessian is meanwhile well understood and has been considered for example in $[6,8,10,13,16,22,26]$. In comparison to [15], we derive here a more precise expansion of the random shape functional. This fact is also verified by our numerical experiments.

This work is organized as follows. In Section 2, we introduce our main theoretical tool: the shape calculus of order two. We define the shape derivative of a general shape function and present the structure. In order to illustrate the method, we choose two examples which we will focus on in this work: the first eigenvalue of the Dirichlet-Laplace operator and the Dirichlet energy. We provide the expressions of the shape derivatives of these two quantities. Then, in Section 3, we detail our random model and explain how one can in general obtain asymptotic expansions of the moments of the quantity of interest's distribution. In Section 4, we emphasize the role of the autocovariance of the field $X$ to compute the previously derived Taylor expansions and explain how to obtain a low-rank approximation of the field $X$ itself. In Section 5, we make explicit the expansions of Section 3 and obtain expressions which are directly workable for computations. Finally, we validate our theoretical findings in Section 6 by comparing our results with Monte-Carlo simulations. 


\section{SECOND ORDER SHAPE CALCUlus}

\subsection{Definitions and structure theorem}

Shape calculus was founded by Hadamard a century ago but was really developed from the seventies on with the works of Murat and Simon as well as Sokolowski and Zolesio. It's objective is to provide a differential calculus for functions which depend on the geometry of a domain $D$. It is achieved through the action of a family of diffeomorphisms acting on a model domain. We refer readers interested in the precise definitions and useful properties to modern books on the subject such as $[9,16]$.

It is well-known since Hadamard's work that, in smooth situations, the shape gradient is a distribution supported on the moving boundary and acting on the normal component of the deformation field that is a vector field defined in $\mathbb{R}^{d}$ with values in $\mathbb{R}^{d}$. In this work, we consider a smooth vector field in $\mathcal{C}^{k, 1}\left(\mathbb{R}^{d}, \mathbb{R}^{d}\right)$, the space of $\mathcal{C}^{k}$-functions with Lipschitz continuous $k$ th derivatives. The second order shape derivative has also a specific structure as stated by Pierre and Novruzi in [22]. We quote their result from the book ([16], Thm. 5-9-2).

Theorem 2.1 (Structure theorem of first and second order shape derivatives). Let $k \geq 1$ be an integer and $J$ a real valued shape function which is defined on $\mathcal{O}_{k}$, the open bounded domains of $\mathbb{R}^{d}$ with a $\mathcal{C}^{k}$-boundary. Let us define the function $\mathcal{J}$ on $\mathcal{C}^{k, 1}\left(\mathbb{R}^{d}, \mathbb{R}^{d}\right)$ by

$$
\mathcal{J}(\theta)=J((I+\theta)(D)) .
$$

(i) If $D \in \mathcal{O}_{k+1}$ and $\mathcal{J}$ is differentiable at 0 , then there exists a continuous linear form $L$ on $\mathcal{C}^{k}(\partial D)$ such that:

$$
D \mathcal{J}(0) \xi=L(\xi \cdot \boldsymbol{n}) \text { for all } \xi \in \mathcal{C}^{k, 1}\left(\mathbb{R}^{d}, \mathbb{R}^{d}\right) .
$$

(ii) If $D \in \mathcal{O}_{k+2}$ and $\mathcal{J}$ is twice differentiable at 0 , then there exists a continuous symmetric bilinear form $B$ on $\mathcal{C}^{k}(\partial D) \times \mathcal{C}^{k}(\partial D)$ such that for all $(\xi, \zeta) \in \mathcal{C}^{k, 1}\left(\mathbb{R}^{d}, \mathbb{R}^{d}\right)^{2}$

$$
D^{2} \mathcal{J}(0)(\xi, \zeta)=B(\xi \cdot \boldsymbol{n}, \zeta \cdot \boldsymbol{n})+L\left(\left(D_{\tau} \boldsymbol{n} \zeta_{\tau}\right) \cdot \xi_{\tau}-\nabla_{\tau}(\zeta \cdot \boldsymbol{n}) \cdot \xi_{\tau}-\nabla_{\tau}(\xi \cdot \boldsymbol{n}) \cdot \zeta_{\tau}\right),
$$

where $\nabla_{\tau}$ is the tangential gradient and $\xi_{\tau}$ and $\zeta_{\tau}$ stands for the tangential components of $\xi$ and $\zeta$. Finally, $D_{\tau} \boldsymbol{n}$ is the differential of the Gauss map known as the shape operator or Weingarten map.

The so-called shape derivatives are then the shape gradient, usually noted $D J(D):=D \mathcal{J}(0)$, and the shape Hessian, usually noted $D^{2} J(D):=D^{2} \mathcal{J}(0)$. With respect to this work, it is important to notice that the shape Hessian is reduced to $B$ for normal deformations fields.

Following the structure theorem, let us consider a $\mathcal{C}^{5}$-domain $D_{0}$ and consider a $\mathcal{C}^{3}$-neighbourhood $\mathcal{O}$ of $D$ and a twice (locally) differentiable shape function $J$. If the size of the neighbourhood is small enough, the local inversion theorem shows that the boundary $\partial D$ of any domain $D$ in $\mathcal{O}$ can be represented as a graph over $\partial D_{0}$ of the form: there is a real valued function $\varphi$ defined on $\partial D_{0}$ such that

$$
\partial D=\left\{x+\varphi(x) \boldsymbol{n}(x) ; x \in \partial D_{0}\right\} .
$$

In particular, one can restrict oneself to normal perturbations of amplitude $\varphi$. Moreover, for a given function $\varphi$ defined on $\partial D_{0}$, the interior of the set $\left\{x+\varphi(x) \boldsymbol{n}(x) ; \quad x \in \partial D_{0}\right\}$ determines a domain $D_{\varphi}$. Thus, the Taylor formula for the mapping $\mathcal{J}$ defined in Theorem 2.1 for $\theta=\varphi \boldsymbol{n}$, where we still have denoted smooth extensions to $\mathbb{R}^{d}$ by $\varphi$ and $\boldsymbol{n}$, reads as

$$
J\left(D_{\varphi}\right)=J\left(D_{0}\right)+L[J]\left(D_{0}\right) \varphi+\frac{1}{2} B[J]\left(D_{0}\right)(\varphi, \varphi)+R_{2}(\varphi) .
$$

Here, the reminder $R_{2}$ is uniformly in $\varphi$ negligible with respect to $\|\varphi\|^{2}$. Note that, in many applications, the shape functions are regular and in particular $\mathcal{C}^{3}$ (see the discussion in [7]). In that case, the remainder is of order 3 in the amplitude $\varepsilon$ of the considered random perturbations. 


\subsection{Examples of shape derivatives}

Shape gradients have been computed for many objectives covering many boundary value problems motivated by applications in civil engineering, inverse problems, aeronautics, etc. A lot of examples can be found in the book [29]. The shape gradient is widely used in level-set methods for shape optimization (see [2] among may others). Due to their much higher complexity, shape Hessians are not so often applied and the literature about computations with second order shape derivatives is restricted (see, e.g., [11,12,14,23]). Nevertheless, the shape Hessian underlies the study of stability issues in shape optimization (see $[1,3,4,6-8,13,21,25,28,31]$ for examples in imaging, tomography, fluid mechanics, aircraft construction, etc.). For the sake of readability, we present two academic but representative examples for which the expressions of the shape derivatives remains simple.

We need to precise some geometrical definitions. The mean curvature (understood as the sum of the principal curvatures of $\partial D$ ) is denoted by $H$. For a domain $D \subset \mathbb{R}^{d}$, we consider its Dirichlet energy $E(D)$ defined as

$$
E(D)=-\frac{1}{2} \int_{D}\left|\nabla u_{D}\right|^{2}
$$

where $u_{D}$ is the solution of $-\Delta u=1$ in $\mathrm{H}_{0}^{1}(D)$ and $\lambda_{1}$ is the first eigenvalue of the Dirichlet-Laplace operator. The shape derivatives of these functionals are well-known (see [16], Sects. 5.9.3 and 5.9.6).

Lemma 2.2 (Expressions of shape derivatives). If $D$ is $\mathcal{C}^{2}$, one has

$$
\begin{aligned}
L[E](D) \cdot \varphi & =-\frac{1}{2} \int_{\partial D}\left(\partial_{n} u\right)^{2} \varphi \\
B[E](D) \cdot(\varphi, \varphi) & =\left\langle-\partial_{n} u \varphi, \Lambda\left(-\partial_{n} u \varphi\right)\right\rangle_{\mathrm{H}^{1 / 2} \times \mathrm{H}^{-1 / 2}}+\int_{\partial D}\left[\partial_{n} u+\frac{1}{2} H\left(\partial_{n} u\right)^{2}\right] \varphi^{2} \\
L\left[\lambda_{1}\right](D) \cdot \varphi & =-\int_{\partial D}\left(\partial_{n} v\right)^{2} \varphi \\
B\left[\lambda_{1}\right](D) \cdot(\varphi, \varphi) & =\int_{\partial D} 2 w(\varphi) \partial_{n} w(\varphi)+H\left(\partial_{n} v\right)^{2} \varphi^{2}
\end{aligned}
$$

where $\Lambda: \mathrm{H}^{1 / 2}(\partial D) \rightarrow \mathrm{H}^{-1 / 2}(\partial D)$ is the Dirichlet-to-Neumann map for the domain $D$ defined as $\Lambda(\varphi)=$ $-\partial_{n} V(\varphi)$ with $V(\varphi)$ being the solution of

$$
-\Delta V(\varphi)=0 \text { in } D, \quad V(\varphi)=-\varphi \text { on } \partial D
$$

and $v$ is the associated normalized eigenfunction solution in $\mathrm{H}_{0}^{1}(D)$ of $-\Delta v=\lambda_{1} v$ with $v>0$ in $D$ and $w(\varphi)$ is the solution of

$$
\left\{\begin{aligned}
-\Delta w(\varphi) & =\lambda_{1} w(\varphi)-v \int_{\partial D}\left(\partial_{n} v\right)^{2} \varphi \text { in } D, \\
w(\varphi) & =-\varphi \partial_{n} v \text { on } \partial D, \\
\int_{D} v w(\varphi) & =0 .
\end{aligned}\right.
$$

As consequence of these examples and being the general case for smooth domains to the best of our knowledge, there is a function $\ell$, defined on $\partial D$, so that the shape gradient can be written as

$$
L(D) \cdot \varphi=\int_{\partial D} \ell \varphi .
$$

This property to be an integral operator with a nice kernel is not true in general for the second order derivative. 


\section{Asymptotic expansions of the moments}

\subsection{The stochastic model: random graphs over $\partial D$}

For modeling the stochastic perturbations $D($.$) of D_{0}$, we introduce a probability space $(\Omega, \mathcal{F}, \mathbb{P})$ and consider stochastic functions $X: \partial D \times \Omega \rightarrow \mathbb{R}$ that define $D(\omega)$ as the interior of the graph $x+X(x, \omega) \mathbf{n}_{\partial D_{0}}(x)$. In order to keep a pertinent geometrical description of sets, this of course requires that the domains $D(\omega)$ remain close to $D_{0}$ at least in the $\mathbb{L}^{\infty}$ sense. In fact, to use the expansion (2.2), we need closeness in the sense of $\mathcal{C}^{2}$. Introducing a small parameter $\varepsilon>0$ and plugging perturbations of the form $x+\varepsilon X(x, \omega) \mathbf{n}_{\partial D_{0}}(x)$ into $(2.2)$, we obtain

$$
J(D(\omega))=J\left(D_{0}\right)+\varepsilon L(X(\omega))+\frac{\varepsilon^{2}}{2} B(X(\omega), X(\omega))+R_{2}(\varepsilon X(\omega)) .
$$

We make the assumption that $X$ is uniformly (in $\omega$ ) bounded in the norm in the spatial variable where shape differentiability holds at the second order (typically here the $\mathcal{C}^{2}$-norm), i.e., $X$ is a member of the Bochner space $\mathbb{L}^{\infty}\left(\Omega, \mathcal{C}^{2}(\partial D)\right)$. Then, there exists $C>0$ such that $\left|R_{2}(\varepsilon X(\omega))\right|=\mathcal{O}\left(\varepsilon^{2}\right)$.

In most applications, the quantity $J(D(\omega))$ is defined through the solution $u_{D(\omega)}$ of a boundary value problem set in $D(\omega)$. Let $B$ be a fixed large ball such that $D(\omega) \subset B$ for all $\omega \in \Omega$. To fix the idea, consider the Dirichlet problem for a second order elliptic partial differential operator $\mathfrak{L}: u_{D}$ is the solution of $\mathfrak{L} u=f$ in $\mathrm{H}_{0}^{1}(D)$. We still denote $u_{D}$ for its extension by 0 to $\mathrm{H}_{0}^{1}(B)$. Let us remark that the mapping $\omega \mapsto u_{D(\omega)}$ is the composition of the mappings $\omega \mapsto X(\omega) \in \mathcal{C}^{2}(\partial D)$ and $X \mapsto u_{D(X)} \in \mathrm{H}_{0}^{1}(B)$ defined through (2.1). Since the second mapping $X \mapsto u_{D(X)} \in \mathrm{H}_{0}^{1}(B)$ is continuous, it is measurable. Moreover, it is strongly measurable by the Orlicz-Pettis theorem since both $\mathcal{C}^{2}(\partial D)$ and $\mathrm{H}_{0}^{1}(B)$ are separable (we used the fact that $D$ is bounded so that $\partial D$ is compact).

\subsection{General preliminary results}

In view of (3.1), we derive the asymptotic expansions of the expectation and variance of $J(D(\omega))$ as our first result. From now, we consider a twice shape differentiable function $J$.

Proposition 3.1. The expectation and variance of $J(D(\omega))$ admit the asymptotic expansions

$$
\mathbb{E}(J(D))=J\left(D_{0}\right)+\varepsilon \mathbb{E}(L(X))+\frac{\varepsilon^{2}}{2} \mathbb{E}(B(X, X))+\mathcal{O}\left(\varepsilon^{2}\right)
$$

and

$$
\begin{aligned}
\operatorname{var}(J(D))= & \varepsilon^{2} \mathbb{E}\left([L(X)-\mathbb{E}(L(X))]^{2}\right) \\
& +\varepsilon^{3} \mathbb{E}([L(X)-\mathbb{E}(L(X))][B(X, X)-\mathbb{E}(B(X, X))])+\mathcal{O}\left(\varepsilon^{3}\right) .
\end{aligned}
$$

Proof of Proposition 3.1. It suffices to integrate the pointwise Taylor expansion (3.1) over the space of probability thanks to the uniform estimate of the remainder. One thus immediately obtains for the expectation

$$
\begin{aligned}
\mathbb{E}(J(D)) & =J\left(D_{0}\right)+\varepsilon \mathbb{E}(L(X))+\frac{\varepsilon^{2}}{2} \mathbb{E}(B(X, X))+\mathbb{E}\left(R_{2}(\varepsilon X)\right) \\
& =J\left(D_{0}\right)+\varepsilon \mathbb{E}(L(X))+\frac{\varepsilon^{2}}{2} \mathbb{E}(B(X, X))+\mathcal{O}\left(\varepsilon^{2}\right) .
\end{aligned}
$$

Likewise, for the variance, one has

$$
\begin{aligned}
\operatorname{var}(J(D)) & =\mathbb{E}\left([J(D)-\mathbb{E}(J(D))]^{2}\right) \\
& =\mathbb{E}\left(\left[\varepsilon(L(X)-\mathbb{E}(L(X)))+\frac{\varepsilon^{2}}{2}(B(X, X)-\mathbb{E}(B(X, X)))+\mathcal{O}\left(\varepsilon^{2}\right)\right]^{2}\right) \\
& =\varepsilon^{2} \mathbb{E}\left([L(X)-\mathbb{E}(L(X))]^{2}\right)+\varepsilon^{3} \mathbb{E}([L(X)-\mathbb{E}(L(X))][B(X, X)-\mathbb{E}(B(X, X))])+\mathcal{O}\left(\varepsilon^{3}\right) .
\end{aligned}
$$


Remark 3.2. Let us notice that it follows $\mathbb{E}(L(X))=L(\mathbb{E}(X))=0$ if $X$ is centered, i.e., if $\mathbb{E}(X)=0$.

In the same spirit as above, we can also compute asymptotic expansions of higher moments. For example, the centered, normalized moments (as skewness and kurtosis) could be obtained in the following way.

Proposition 3.3. For all $k \geq 2$, the centered normalized moment of $J(D(\omega))$ admits the asymptotic expansion

$$
\mathbb{M}_{k}(J(D)):=\mathbb{E}\left(\frac{[J(D)-\mathbb{E}(J(D))]^{k}}{\sqrt{\operatorname{var}(J(D))}^{k}}\right)=a_{k}+b_{k} \varepsilon+\mathcal{O}(\varepsilon),
$$

where the deterministic coefficients $a_{k}$ and $b_{k}$ are

$$
\begin{gathered}
a_{k}=\gamma_{k} \mathbb{E}\left([L(X)-\mathbb{E}(L(X))]^{k}\right) \mathbb{E}\left([L(X)-\mathbb{E}(L(X))]^{2}\right), \\
b_{k}=\gamma_{k} \frac{k}{2}\left\{\mathbb{E}\left([L(X)-\mathbb{E}(L(X))]^{k-1}[B(X, X)-\mathbb{E}(B(X, X))]\right) \mathbb{E}\left([L(X)-\mathbb{E}(L(X))]^{2}\right)\right. \\
\left.-\mathbb{E}([L(X)-\mathbb{E}(L(X))][B(X, X)-\mathbb{E}(B(X, X))]) \mathbb{E}\left([L(X)-\mathbb{E}(L(X))]^{k}\right)\right\},
\end{gathered}
$$

and the normalization constant $\gamma_{k}$ is

$$
\gamma_{k}=\mathbb{E}\left([L(X)-\mathbb{E}(L(X))]^{2}\right)^{-1-k / 2} .
$$

Proof of Proposition 3.3. The second order Taylor expansion of the real function $x \mapsto x^{-k / 2}$ around a real number $x$ is

$$
\frac{1}{\sqrt{x+h}^{k}}=\frac{1}{\sqrt{x}^{k}}-\frac{h k}{2 \sqrt{x}^{k+2}}+\mathcal{O}\left(h^{2}\right) .
$$

Inserting the expansion of the variance (3.3), we find

$$
\begin{aligned}
\frac{1}{\sqrt{\operatorname{var}(J(D))}}= & \frac{1}{\varepsilon^{k}}\left\{\mathbb{E}\left([L(X)-\mathbb{E}(L(X))]^{2}\right)^{-k / 2}\right. \\
& -\frac{\varepsilon k}{2} \mathbb{E}\left(\left[L(X)-\mathbb{E}(L(X)]^{2}\right)^{-1-k / 2} \mathbb{E}([L(X)-\mathbb{E}(L(X))][B(X, X)-\mathbb{E}(B(X, X))])\right. \\
& \left.+\mathcal{O}\left(\varepsilon^{2}\right)\right\} .
\end{aligned}
$$

On the other hand, using the expansion of the expectation (3.2), we have

$$
\begin{aligned}
\mathbb{E}\left([J(D)-\mathbb{E}(J(D))]^{k}\right)= & \varepsilon^{k}\left\{\mathbb{E}\left([L(X)-\mathbb{E}(L(X))]^{k}\right)\right) \\
& \left.+\frac{\varepsilon k}{2} \mathbb{E}\left([L(X)-\mathbb{E}(L(X))]^{k-1}[B(X, X)-\mathbb{E}(B(X, X))]\right)+\mathcal{O}\left(\varepsilon^{2}\right)\right\} .
\end{aligned}
$$

Then, a tedious but elementary calculus (the product of two asymptotic expansions) leads to the desired result. 


\section{Efficient computation of the Taylor coefficients - The general Case}

\subsection{Direct computation}

For the sake of simplicity, we shall assume that the boundary perturbation field $X$ is centered which induces $\mathbb{E}(L(X))=0$. We then can compute as in $[15]$

$$
\begin{aligned}
\mathbb{E}\left([L(X)-\mathbb{E}(L(X))]^{2}\right) & =\mathbb{E}\left(L(X)^{2}\right)=\mathbb{E}\left[\left(\int_{\partial D} \ell(x) X(x, \omega) \mathrm{d} \sigma(x)\right)^{2}\right] \\
& =\mathbb{E}\left[\int_{\partial D} \int_{\partial D} X(x, \omega) X(y, \omega) \ell(x) \ell(y) \mathrm{d} \sigma(x) \mathrm{d} \sigma(y)\right] \\
& =\int_{\partial D} \int_{\partial D} \mathbb{E}[X(x, .) X(y, .)] \ell(x) \ell(y) \mathrm{d} \sigma(x) \mathrm{d} \sigma(y) .
\end{aligned}
$$

The crucial operator to be studied here is the (two-point) autocovariance function of $X$ defined as

$$
\operatorname{Cov}_{X}(x, y)=\mathbb{E}(X(x, .) X(y, .)) .
$$

In fact, the knowledge of the law of $X$ is not needed for the computation we have in mind, we only need the autocovariance function. Notice that various laws for $X$ can provide the same function $\operatorname{Cov}_{X}$.

We can proceed in two different ways to compute the expectation of the shape Hessian. The first approach has already been pointed out in e.g. [20]. We shall explain the proceeding in case of the Dirichlet energy. In that case, according to $(2.3 \mathrm{~b})$, we have

$$
\mathbb{E}(B(X, X))=\mathbb{E}\left(\int_{\partial D}\left[\partial_{n} u+\frac{1}{2} H\left(\partial_{n} u\right)^{2}\right] X^{2} \mathrm{~d} \sigma\right)+\mathbb{E}\left(\left\langle-\partial_{n} u X, \Lambda\left(-\partial_{n} u X\right)\right\rangle_{\mathrm{H}^{1 / 2} \times \mathrm{H}^{-1 / 2}}\right) .
$$

For the first term, one has easily by Fubini's theorem that

$$
\begin{aligned}
\mathbb{E}\left(\int_{\partial D}\left[\partial_{n} u+\frac{1}{2} H\left(\partial_{n} u\right)^{2}\right] X^{2} \mathrm{~d} \sigma\right) & =\int_{\partial D} \mathbb{E}\left(X^{2}\right)\left[\partial_{n} u+\frac{1}{2} H\left(\partial_{n} u\right)^{2}\right] \mathrm{d} \sigma \\
& =\int_{\partial D} \mathbb{V}(X)\left[\partial_{n} u+\frac{1}{2} H\left(\partial_{n} u\right)^{2}\right] \mathrm{d} \sigma
\end{aligned}
$$

since $X$ is centered. To compute the second term, we shall define the following tensor product type boundary value problem:

$$
\begin{aligned}
(i d \otimes(-\Delta)) V & =0 & & \text { in } \partial D \times D, \\
V & =\left(i d \otimes\left(-\partial_{n} u\right)\right) \operatorname{Cov}_{X} & & \text { on } \partial D \times \partial D .
\end{aligned}
$$

Here, $-\partial_{n} u$ has to be understood in the sense of a multiplication operator $\mathrm{H}^{1 / 2}(\partial D) \rightarrow H^{1 / 2}(\partial D)$. Note that the differential operator which underlies this boundary value problem is $i d \otimes \Lambda$. Due to its linearity and Fubini's theorem, we get thus for the second term

$$
\begin{aligned}
\mathbb{E}\left(\left\langle-\partial_{n} u X, \Lambda\left(-\partial_{n} u X\right)\right\rangle_{\mathrm{H}^{1 / 2} \times \mathrm{H}^{-1 / 2}}\right) & =\left.\int_{\partial D} \mathbb{E}\left(-\partial_{n} u(x) X(x, \cdot)\left(\Lambda\left(-\partial_{n} u X\right)\right)(y, \cdot)\right)\right|_{x=y} \mathrm{~d} \sigma(x) \\
& =\int_{\partial D}-\left.\partial_{n} u(x) V(x, y)\right|_{x=y} \mathrm{~d} \sigma(x) .
\end{aligned}
$$

Boundary value problems like that in (4.1) can be solved in essentially linear complexity (with respect to the number of unknowns to discretize the state equation on the domain $D$ ) if a sparse tensor product discretization is employed as proposed in e.g. $[17,19,20]$. However, the implementation of this approach would be highly intrusive. 


\subsection{Toward a low-rank approximation}

The second way, which is much simpler to implement, consists in computing an expansion of the autocovariance function of $X$ of the form

$$
\operatorname{Cov}_{X}=\sum_{k} \kappa_{k} \otimes \kappa_{k}
$$

For example, such a representation can be achieved by a spectral decomposition as an application of Mercer's theorem which ensures the representation of $\operatorname{Cov}_{X}$ in the form

$$
\operatorname{Cov}_{X}(x, y)=\sum_{k} \lambda_{k} e_{k}(x) e_{k}(y)
$$

Another way to obtain such a decomposition is an (possibly infinite) Cholesky decomposition of autocovariance function. With the expansion (4.2) at hand, we will obtain in Section 5 the following expansion of the expectation of the shape Hessian:

$$
\mathbb{E}(B[J](D) \cdot(X, X))=\sum_{k} B[J](D) \cdot\left(\kappa_{k}, \kappa_{k}\right) .
$$

We therefore need only to be able to evaluate the shape Hessian in certain directions $\left\{\kappa_{i}\right\}$.

\subsection{Numerical realization}

In general, the expansion (4.2) will be infinite and has to be appropriately truncated for numerical computations. Consider a suitable ansatz space $V_{n}=\operatorname{span}\left\{\varphi_{i}: i=1,2, \ldots, n\right\} \subset \mathcal{C}^{2,1}(\partial D)$. Then, we aim at a low-rank approximation

$$
\operatorname{Cov}_{X}(x, y) \approx \sum_{k=1}^{m}\left(\sum_{i=1}^{n} \ell_{k, i} \varphi_{i}(x)\right)\left(\sum_{j=1}^{n} \ell_{k, j} \varphi_{j}(y)\right) \in V_{n} \otimes V_{n}
$$

to the two-point autocovariance $\operatorname{Cov}_{X} \in L^{2}(\partial D \times \partial D)$ in the tensor product space $V_{n} \otimes V_{n}$.

The unknown coefficient vectors in (4.4) can be computed as follows. Define the discrete autocovariance matrix

$$
\mathbf{C}=\left[\int_{\partial D} \int_{\partial D} \operatorname{Cov}_{X}(x, y) \varphi_{i}(x) \varphi_{j}(y) \mathrm{d} \sigma(x) \mathrm{d} \sigma(y)\right]_{i, j} \in \mathbb{R}^{n \times n}
$$

and the mass matrix

$$
\mathbf{G}=\left[\int_{\partial D} \varphi_{i}(x) \varphi_{j}(x) \mathrm{d} \sigma(x)\right]_{i, j} \in \mathbb{R}^{n \times n}
$$

with respect to the ansatz space $V_{n}$. Then, (4.4) amounts to an approximation

$$
\mathbf{C} \approx \mathbf{C}_{m}=\sum_{k=1}^{m} \tilde{\boldsymbol{\ell}}_{k} \tilde{\ell}_{k}^{T} \quad \text { with } \quad \boldsymbol{\ell}_{k}=\left[\ell_{k, i}\right]_{i}=\mathbf{G}^{-1} \widetilde{\ell}_{k}
$$

such that the truncation error $\left\|\mathbf{C}-\mathbf{C}_{m}\right\|$ is rigorously controllable.

The best low-rank approximation in $L^{2}(\partial D \times \partial D)$ is given by truncating the spectral decomposition (4.3). The computation requires the knowledge of the eigenpairs $\left(\varphi_{i}, \lambda_{i}\right)$ of the integral operator

$$
(\mathcal{C} u)(x):=\int_{\partial D} \operatorname{Cov}_{X}(x, y) u(y) \mathrm{d} \sigma(y), \quad x \in \partial D
$$

which is a very demanding task. In particular, the decay of the eigenvalues $\left\{\lambda_{k}\right\}$ and thus the rank $m$ depend heavily on the smoothness of the autocovariance function $\operatorname{Cov}_{X}$. Related decay rates have been proven in [27]. 
We suggest using the pivoted Cholesky decomposition to compute a low-rank approximation of $\operatorname{Cov}_{X}$ as proposed in [18]. It is a purely algebraic approach which is quite simple to implement, see e.g. [18]. It produces a low-rank approximation to $\mathbf{C}$ for any given precision $\varepsilon>0$ where the approximation error is rigorously controlled in the trace norm. A rank- $m$ approximation is computed in $\mathcal{O}\left(m^{2} n\right)$ operations. Exponential convergence rates in $m$ are proven under the assumption that the eigenvalues of $\mathbf{C}$ exhibit a sufficiently fast exponential decay, see [18]. Nevertheless, numerical experiments show that, in general, the pivoted Cholesky decomposition converges optimally in the sense that the rank $m$ is uniformly bounded with respect to the truncation error $\varepsilon$ by the number of terms required for the spectral decomposition of $\operatorname{Cov}_{X}$ to get the same error $\varepsilon$.

\section{Computing the Coefficients For A LOW-RAnK Approximation of $X$}

Let us apply Proposition 3.1 which provides the Taylor expansion of shape functionals. To end the, let $\left(f_{i}\right)_{i \in \mathbb{N}}$ be a sequence of smooth (at least $\mathcal{C}^{2,1}$ ) real valued functions which are defined on $\partial D$, possibly with compact support on $\partial D$ if needed, and such that the series $\sum_{i \in \mathbb{N}}\left\|f_{i}\right\|_{\mathcal{C}^{2,1}}$ is convergent. We consider the domains defined by their boundary

$$
\partial D_{\varepsilon}(\omega)=\{x+\varepsilon X(x, \omega) \boldsymbol{n}(x) ; X \in \partial D\}
$$

with

$$
X(x, \omega)=\sum_{i=1}^{\infty} \alpha_{i}(\omega) f_{i}(x)
$$

where the $\alpha_{i}$ are either uncorrelated or independent and identically distributed following the same centered distribution $\mathcal{L}$ of a random variable $\alpha$ with finite second order moment. Note that such a model can also be provided by the Karhunen-Loève expansion, even though the $f_{i}$, given by Mercer's theorem, are only in $\mathrm{L}^{2}(\partial D)$ and the additionally required regularity has to be checked, see e.g. [27].

Proposition 5.1. When the random graph has the form (5.1) with uncorrelated and centered random coefficients $\left(\alpha_{i}\right)$, one has

$$
\mathbb{E}(J(D))=J\left(D_{0}\right)+\frac{\varepsilon^{2}}{2} \mathbb{E}\left(\alpha^{2}\right) \sum_{i=1}^{\infty} B\left(f_{i}, f_{i}\right)+\mathcal{O}\left(\varepsilon^{2}\right) .
$$

If the random coefficients are independent and if the distribution $\alpha$ has finite moments up to the order 3 , then

$$
\operatorname{var}(J(D))=\varepsilon^{2} \mathbb{E}\left(\alpha^{2}\right) \sum_{i=1}^{\infty}\left(L\left(f_{i}\right)\right)^{2}+\varepsilon^{3} \mathbb{E}\left(\alpha^{3}\right) \sum_{i=1}^{\infty} L\left(f_{i}\right) B\left(f_{i}, f_{i}\right)+\mathcal{O}\left(\varepsilon^{3}\right) .
$$

Moreover, if the random variable $\alpha$ is symmetric, it even holds

$$
\operatorname{var}(J(D))=\varepsilon^{2} \mathbb{E}\left(\alpha^{2}\right) \sum_{i=1}^{\infty}\left(L\left(f_{i}\right)\right)^{2}+\mathcal{O}\left(\varepsilon^{3}\right) .
$$

Proof of Proposition 5.1. We first notice that, for any integer $k \geq 1$, the series $\sum_{i \in \mathbb{N}}\left\|f_{i}\right\|_{\mathcal{C}^{2,1}}^{k}$ is convergent. 1. Expansion of the expectation. By linearity, it comes directly

$$
\mathbb{E}(L(X))=L(\mathbb{E}(X))=0 .
$$

For any integer $n$, we introduce the truncated series $X_{n}$ defined as:

$$
X_{n}(x, \omega)=\sum_{i=1}^{n} \alpha_{i}(\omega) f_{i}(x)
$$


so that

$$
\mathbb{E}\left(B\left(X_{n}, X_{n}\right)\right)=\mathbb{E}\left(B\left(\sum_{i=1}^{n} \alpha_{i} f_{i}, \sum_{i=1}^{n} \alpha_{i} f_{i}\right)\right)=\sum_{i, j=1}^{n} \mathbb{E}\left(\alpha_{i} \alpha_{j}\right) B\left(f_{i}, f_{j}\right)=\sum_{i=1}^{n} \mathbb{E}\left(\alpha^{2}\right) B\left(f_{i}, f_{i}\right)
$$

since $B$ is bilinear and the $\left(\alpha_{i}\right)$ are uncorrelated and centered. Since $B$ is continuous, we get $\left|B\left(f_{i}, f_{i}\right)\right| \leq$ $\|B\|\left\|f_{i}\right\|_{\mathcal{C}^{2}}^{2}$ and the series $\sum_{i=1}^{\infty} B\left(f_{i}, f_{i}\right)$ converges, so that by continuity and Lebesgue's dominated convergence theorem

$$
\mathbb{E}(B(X, X))=\sum_{i=1}^{\infty} \mathbb{E}\left(\alpha^{2}\right) B\left(f_{i}, f_{i}\right) .
$$

2. Expansion of the variance. We proceed as before for the second order term:

$$
\mathbb{E}\left(\left[L\left(X_{n}\right)-\mathbb{E}\left(L\left(X_{n}\right)\right)\right]^{2}\right)=\mathbb{E}\left(L\left(\sum_{i=1}^{n} \alpha_{i} f_{i}\right)^{2}\right)=\sum_{i, j=1}^{n} \mathbb{E}\left(\alpha_{i} \alpha_{j}\right) L\left(f_{i}\right) L\left(f_{j}\right)=\sum_{i=1}^{n} \mathbb{E}\left(\alpha^{2}\right) L\left(f_{i}\right)^{2} .
$$

We conclude by using the continuity of the map $L$ that

$$
\mathbb{E}\left([L(X)-\mathbb{E}(L(X))]^{2}\right)=\sum_{i=1}^{\infty} \mathbb{E}\left(\alpha^{2}\right) L\left(f_{i}\right)^{2} .
$$

For the third order term, one thus has

$$
\begin{aligned}
& \left.\mathbb{E}\left(\left[L\left(X_{n}\right)-\mathbb{E}\left(L\left(X_{n}\right)\right)\right]\left[B\left(X_{n}, X_{n}\right)-\mathbb{E}\left(B\left(X_{n}, X_{n}\right)\right)\right)\right]\right)=\mathbb{E}\left(L\left(\sum_{i=1}^{n} \alpha_{i} f_{i}\right) B\left(\sum_{i=1}^{n} \alpha_{i} f_{i}, \sum_{i=1}^{n} \alpha_{i} f_{i}\right)\right) \\
& =\sum_{i, j, k=1}^{n} \mathbb{E}\left(\alpha_{i} \alpha_{j} \alpha_{k}\right) L\left(f_{i}\right) B\left(f_{j}, f_{k}\right)=\sum_{i=1}^{n} \mathbb{E}\left(\alpha^{3}\right) L\left(f_{i}\right) B\left(f_{i}, f_{i}\right)
\end{aligned}
$$

where we used that the distribution of the random variable $\alpha$ is centered.

Remark 5.2. For all $k \geq 2$, if the distribution $\alpha$ has finite moments up to the order $k+1$, then the normalized, centered moment of $J(D(\omega))$ admits the asymptotic expansion

$$
\mathbb{M}_{k}(J(D))=a_{k}+b_{k} \varepsilon+\mathcal{O}(\varepsilon)
$$

where the deterministic coefficients $a_{k}$ and $b_{k}$ could be obtained by a similar tedious but easy calculus. The crucial point is the computation of $\mathbb{E}\left(\Pi_{j=1}^{k} \alpha_{i_{j}}\right)$ for $i_{j} \in\{1, \ldots, N\}$ with the help of the multinomial formula. Thanks to the independence of the $\alpha_{i}$ and the fact that they follow a centered distribution, the previous expression cancels as soon as there is an integer $i \in\{1, \ldots, N\}$ that is reached only once, i.e., if the equation $i=i_{j}$ for $1 \leq j \leq k$ has only a single solution.

For instance, for $k=3$, it holds

$$
\mathbb{M}_{3}(J(D)):=a_{3}+b_{3} \varepsilon+\mathcal{O}(\varepsilon)
$$

with

$$
\begin{gathered}
a_{3}=\frac{\mathbb{E}\left(\alpha^{3}\right)}{\gamma^{3 / 2}} \sum_{i=1}^{\infty} L\left(f_{i}\right)^{3} \\
b_{3}=\frac{3}{2 \gamma^{3 / 2}}\left[\mathbb{E}\left(\alpha^{2}\right)^{2}\left(\sum_{i \neq j} L\left(f_{i}\right) L\left(f_{j}\right) B\left(f_{i}, f_{j}\right)-\sum_{i=1}^{\infty} L\left(f_{i}\right)^{2} B\left(f_{i}, f_{i}\right)\right)+\mathbb{E}\left(\alpha^{4}\right) \sum_{i=1}^{\infty} L\left(f_{i}\right)^{2} B\left(f_{i}, f_{i}\right)\right] \\
-\frac{3}{2 \gamma^{5 / 2}} \mathbb{E}\left(\alpha^{3}\right)^{2} \sum_{i=1}^{\infty} L\left(f_{i}\right) B\left(f_{i}, f_{i}\right) \sum_{i=1}^{\infty} L\left(f_{i}\right)^{3}
\end{gathered}
$$


where

$$
\gamma=\mathbb{E}\left(\alpha^{2}\right) \sum_{i=1}^{\infty} L\left(f_{i}\right)^{2}
$$

With the help of the pivoted Cholesky decomposition, we can compute a low-rank approximation of the random field $X$ which corresponds to a finite sum of the type

$$
X(x, \omega)=\sum_{i=1}^{N} \alpha_{i}(\omega) f_{i}(x)
$$

where the $\alpha_{i}$ are either uncorrelated or independent and identically distributed following the same centered distribution $\mathcal{L}$ of a random variable $\alpha$. In that case, the Taylor coefficients can very easily be computed in accordance with Proposition 5.1: the computational cost reduces to the solution of $N+1$ boundary values problems defined on the same domain so that a single mesh can be used. This amount is doubled to $2 N+2$ resolutions in case of general shape functionals where also an adjoint state has to be computed to efficiently evaluate the shape derivatives (see [16], Sects. 5-8 for an example of the adjoint state in shape optimization).

Finally, using the Bienaymé-Chebychev inequality, we obtain intervals in which the shape functional takes its values with a fixed probability. For example, fixing a desired probability $p$, we get the bounds

$$
\mathbb{P}\left(|J(D)-\mathbb{E}(J(D))| \leq \sqrt{\frac{\operatorname{var}(J(D))}{1-p}}\right) \geq p .
$$

Thanks to the asymptotic expansions (5.3)-(5.4), we obtain then

$$
\mathbb{P}\left(J_{\varepsilon, p}^{-}(D) \leq J(D) \leq J_{\varepsilon, p}^{+}(D)\right) \geq p
$$

where we have set

$$
\begin{aligned}
J_{\varepsilon, p}^{ \pm}(D)=J\left(D_{0}\right) & \pm \frac{\varepsilon}{\sqrt{1-p}} \sqrt{\mathbb{E}\left(\alpha^{2}\right) \sum_{i=1}^{N}\left(L\left(f_{i}\right)\right)^{2}} \\
& +\frac{\varepsilon^{2}}{2}\left(\mathbb{E}\left(\alpha^{2}\right) \sum_{i=1}^{N} B\left(f_{i}, f_{i}\right) \pm \frac{\mathbb{E}\left(\alpha^{3}\right)}{\sqrt{1-p}} \frac{\sum_{i=1}^{N} L\left(f_{i}\right) B\left(f_{i}, f_{i}\right)}{\sqrt{\mathbb{E}\left(\alpha^{2}\right) \sum_{i=1}^{N}\left(L\left(f_{i}\right)\right)^{2}}}\right)+\mathcal{O}\left(\varepsilon^{2}\right) .
\end{aligned}
$$

\section{NumERICAL ILLUSTRATIONS}

We now present numerical validations of the proposed asymptotic expansions. We will consider the twodimensional case for the Dirichlet energy and the first eigenvalue of the Dirichlet-Laplace operator. Numerical resolution of the boundary values problems is made either with the finite element method or with the boundary element method. We will consider both, the uniform and the Beta distribution. During the Monte-Carlo simulations, the random number generator has been reinitialized after at most 10000 samples.

\subsection{The Dirichlet energy around disk in dimension two}

\subsubsection{Random perturbations defined by a given spectral representation}

We consider the unit disk in the plane and random perturbations of the type

$$
D_{\varepsilon}(\omega)=\left\{(r, \theta): 0 \leq r<1+\varepsilon f(\theta, \omega) \text { and } f(\theta)=\sum_{i=1}^{N} \alpha_{i}(\omega) f_{i}(\theta)\right\}
$$




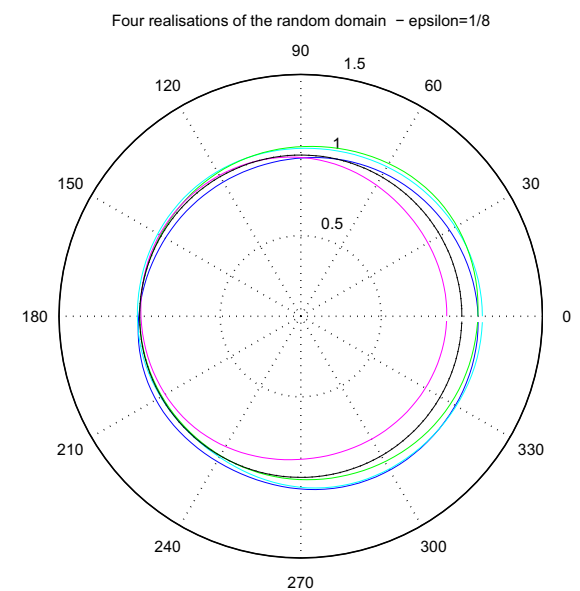

(a) $\varepsilon=1 / 8$.

FIGURE 1. Some realizations of domains according to (6.1) with the uniform distribution.

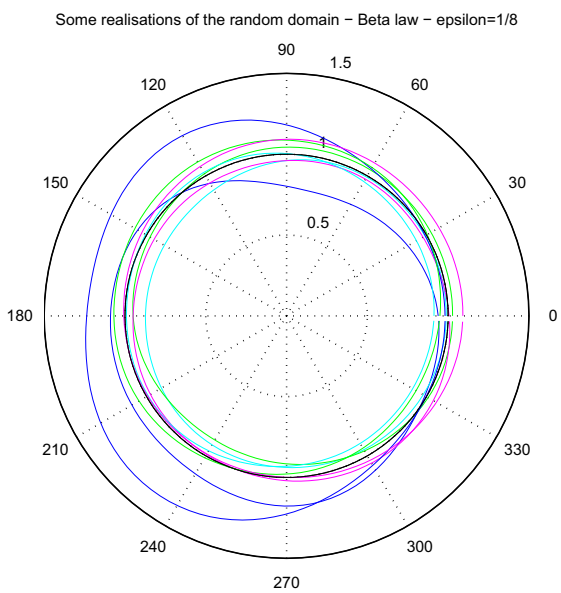

(a) $\varepsilon=1 / 8$.

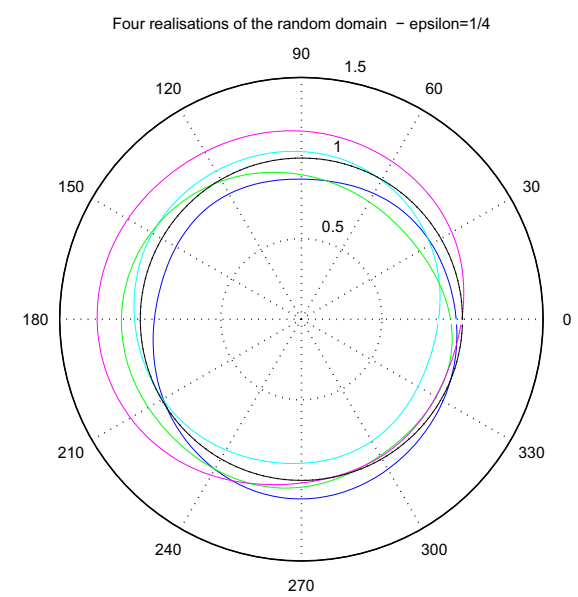

(b) $\varepsilon=1 / 4$.

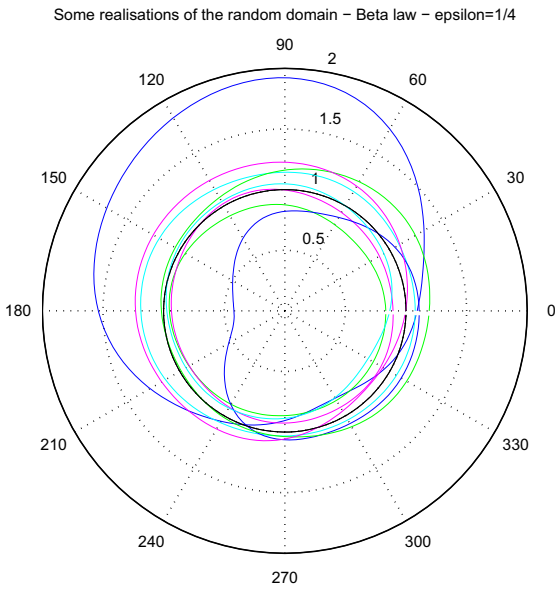

(b) $\varepsilon=1 / 4$.

FiguRE 2. Some realizations of domains according to (6.1) with the Beta distribution.

where the $\alpha_{i}$ are i.i.d. In the following computations, we have taken $N=11$ and the coefficient functions $f_{i}$ are the first normalized functions in the Fourier basis, that is

$$
f_{1}=1, f_{2}(\theta)=\cos (\theta), f_{3}(\theta)=\sin (\theta), f_{4}(\theta)=\cos (2 \theta) / 4, f_{5}(\theta)=\sin (2 \theta) / 4, \ldots, f_{11}(\theta)=\sin (5 \theta) / 25 .
$$

Let us present some realizations of such domains with various values of $\varepsilon$ and various distributions. In Figure 1 , we present some realizations of such random domains for $\alpha$ following the uniform distribution on $[-1 / 2,1 / 2]$. Note that the range of $\alpha$ is $[-1 / 2,1 / 2]$.

In Figure 2, $\alpha$ follows the centered and normalized Beta distribution of parameter $(2,5)$ in order to use a non-symmetric distribution. Note that the range of this distribution is larger (it can take values greater than 4). Hence, for the same value of the parameter $\varepsilon$, the perturbations can be wider for the Beta distribution than for the uniform distribution. Therefore, we will consider a smaller range of values for the parameter $\varepsilon$. 


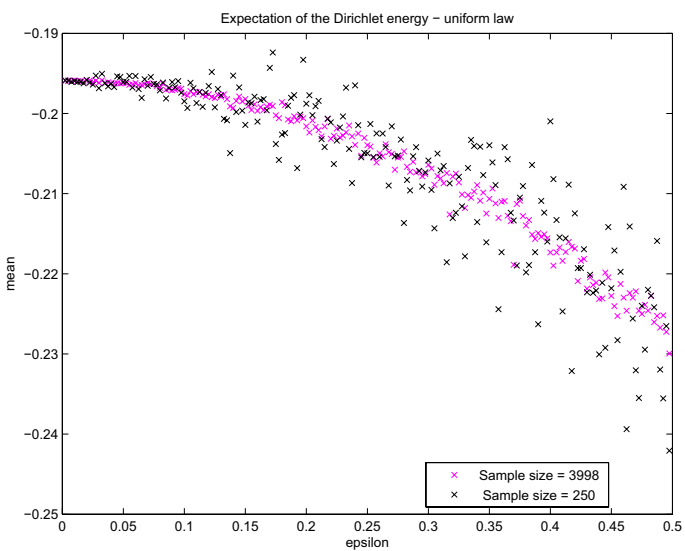

(a) Expectation

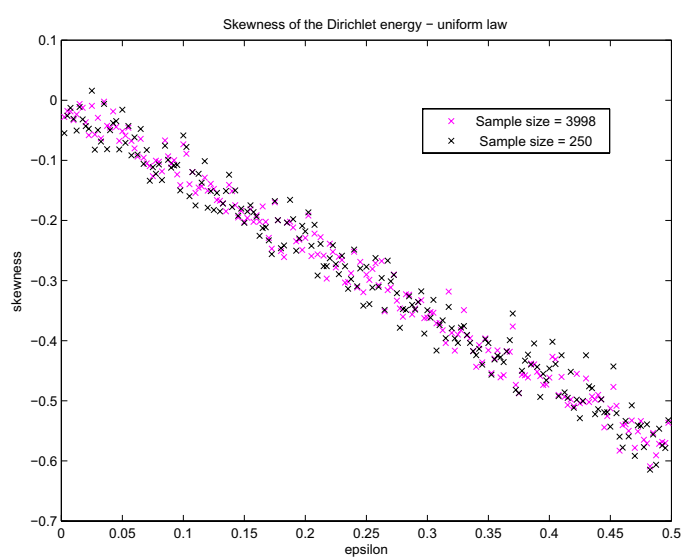

(c) Skewness

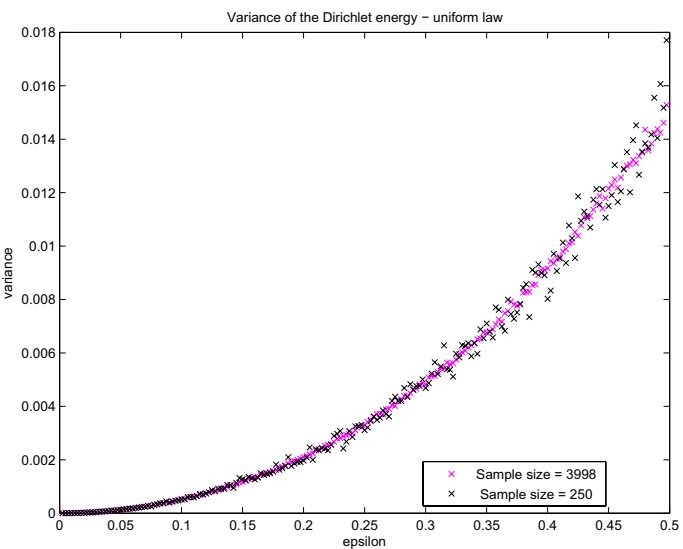

(b) Variance

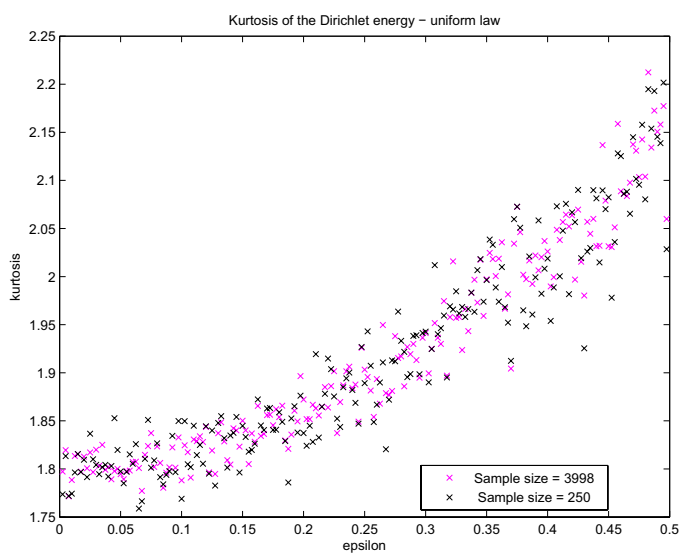

(d) Kurtosis

FiguRE 3. Convergence of Monte-Carlo simulations for perturbed disks defined in (6.1).

\subsubsection{Monte-Carlo simulations}

We shall proceed with the Monte-Carlo method. For each value of $\varepsilon$ in the abscissa of the graphs, we perform 4000 simulations using $P 1$ finite elements over around 2000 triangles, each of which is made with the FREEFEM ++ code. The convergence is illustrated in Figure 3. It seems that a visually correct asymptotic regime is reached for some thousands of simulations.

\subsubsection{Comparison with the asymptotic expansions}

Finally, we compare with the asymptotic expansions obtained in Proposition 5.1. Let us emphasize that the coefficients appearing in Proposition 5.1 are computed numerically. The first step is to compute the state equation in the reference domain, then the shape gradient is obtained via the computation of an integral over the boundary. Finally, the computation of each term $B\left(f_{i}, f_{i}\right)$ requires the solution of a boundary value problem of the same type than the one solved for the state equation. Therefore, the computation of these coefficients is cheap since it requires only $N+1$ resolutions of a boundary value problem. The results for both, the uniform distribution and the Beta distribution, are presented in Figure 4. 


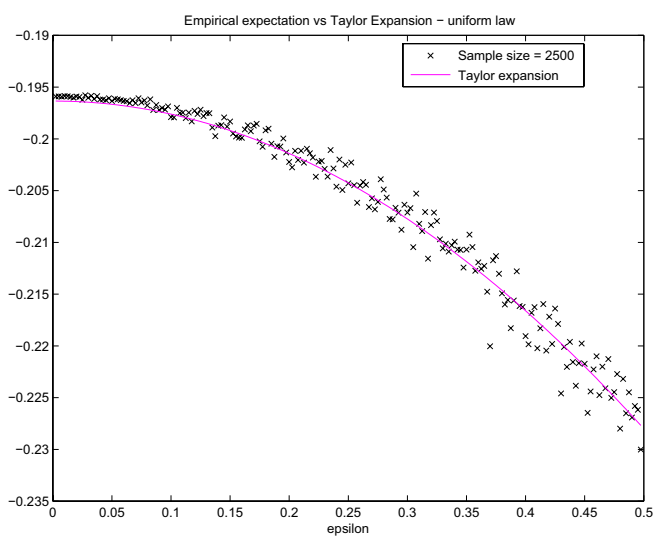

(a) Expectation

Uniform distribution

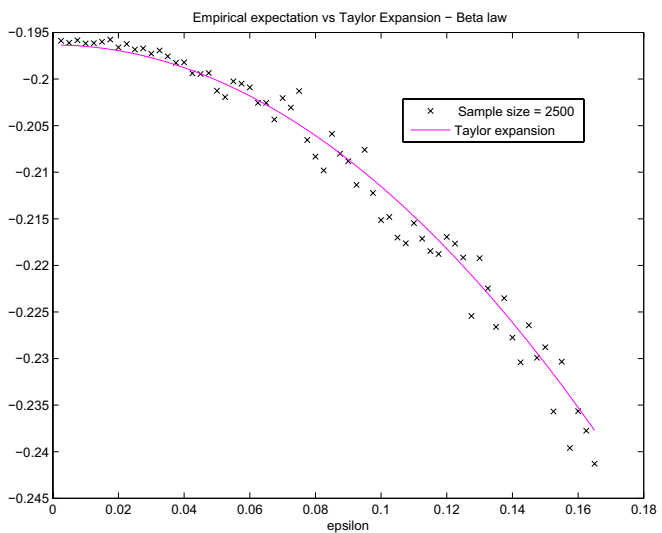

(c) Expectation

Beta distribution

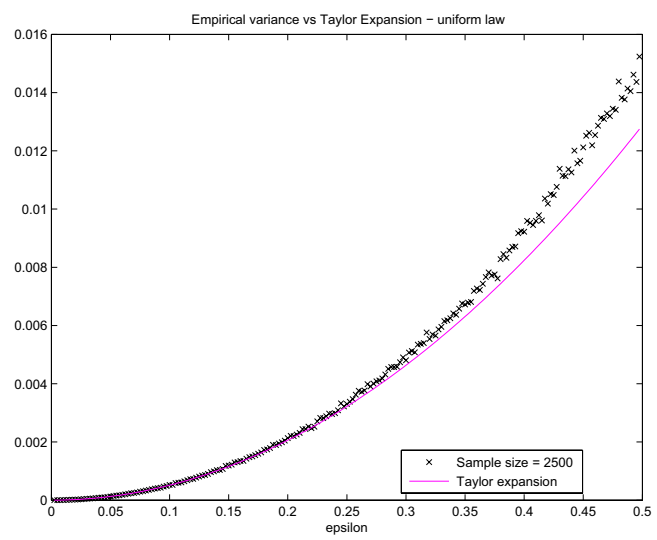

(b) Variance

Uniform distribution

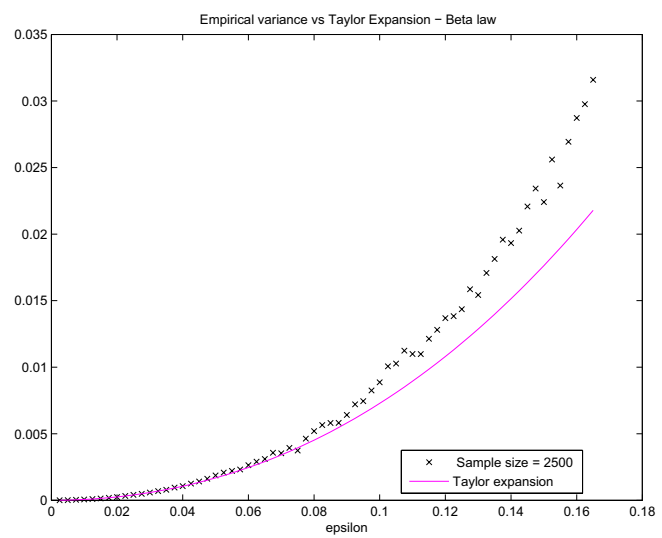

(d) Variance

Beta distribution

Figure 4. Comparison of the Monte-Carlo simulation with the asymptotic expansions for perturbed disks defined in (6.1).

We can also use (5.6) to obtain bounds of intervals of values taken by the Dirichlet energy. In Figure 5 , we have plot both, the graphs of the functions $J_{\varepsilon, p}^{ \pm}$and the empirical quantiles. Notice that the bounds obtained by the asymptotic expansions of the expectation and the variance are better than expected. Such a nice behavior may depend on the specific choice of the Fourier basis, since the shape gradient and the shape Hessian evaluated at the $f_{i}$ decrease fast as shown by the following computed values:

$$
\begin{array}{rlrl}
L\left(f_{1}\right) & =-0.786069 & B\left(f_{1}, f_{1}\right) & =-2.35621 \\
L\left(f_{2}\right) & =-1.74702 \times 10^{-6} & B\left(f_{2}, f_{2}\right) & =-0.392049 \\
L\left(f_{4}\right) & =4.71636 \times 10^{-6} & B\left(f_{4}, f_{4}\right) & =0.0246288 \\
L\left(f_{6}\right) & =3.00416 \times 10^{-7} & B\left(f_{6}, f_{6}\right) & =0.0145671 \\
L\left(f_{8}\right) & =4.24149 \times 10^{-8} & B\left(f_{8}, f_{8}\right) & =0.00767894 \\
L\left(f_{10}\right) & =-1.48682 \times 10^{-7} & B\left(f_{10}, f_{10}\right) & =0.00440313 .
\end{array}
$$




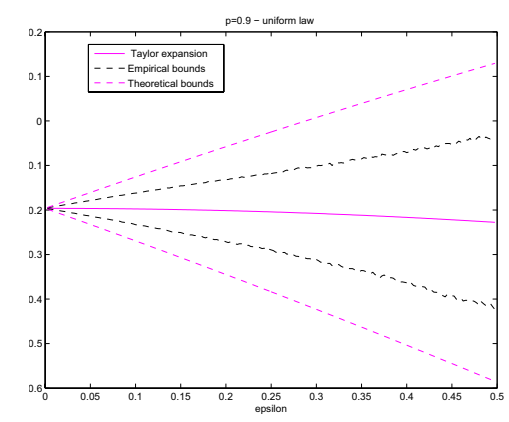

(a) $p=0.9$

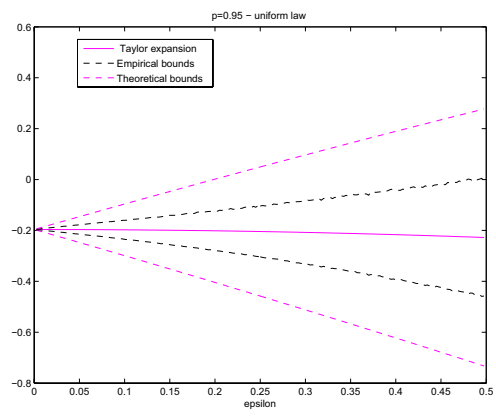

(b) $p=0.95$

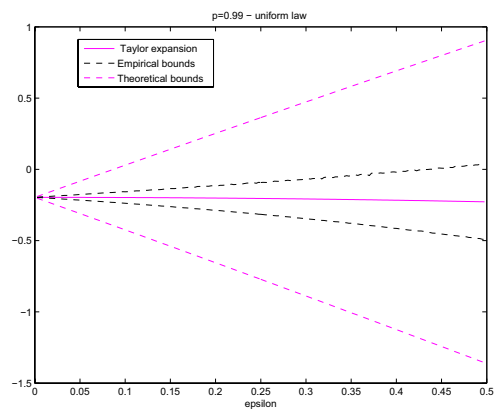

(c) $p=0.99$

Figure 5. Comparison between asymptotic expansions' bounds and empirical quantiles for perturbed disks defined in (6.1).

\subsubsection{Random perturbations known by their mean and covariance}

In this second test, we change the type of random perturbations: instead of being decomposed with respect to the Fourier basis, $X(\omega)$ is now described by its zero mean and its covariance function. We shall consider models with standard covariance kernels, namely Mátern kernels of smoothness class $\nu$. Specifically, we choose $\nu=\infty$ which yields the Gaussian kernel $k_{\infty}(x, y)=\exp \left(-|x-y|^{2} / 2\right)$, we choose $\nu=1 / 2$ which yields the exponential kernel $k_{1 / 2}(x, y)=\exp (-|x-y|)$, and we choose $\nu=3 / 2$ which yields the kernel $k_{3 / 2}(x, y)=$ $(1-\sqrt{3}|x-y|) \exp (-\sqrt{3}|x-y|)$. The covariance kernels are discretized by 100 periodic cubic B-splines on an equidistant subdivision of $[0,2 \pi]$ in accordance with Section 4.3. The pivoted Cholesky decomposition with accuracy $10^{-6}$ yields a rank of $m=19$ for $k_{\infty}$, a rank of 69 for $k_{3 / 2}$, and a rank of $m=100$ for $k_{1 / 2}$.

We still compare a Monte-Carlo simulation with the asymptotic expansions derived in this work for different values $\varepsilon \leq 0.2$. For the Monte-Carlo simulation, the truncated Karhunen-Lóeve expansion is exploited:

$$
X(\omega, \theta)=\sum_{i=0}^{m} \alpha_{i}(\omega) \sqrt{\lambda_{i}} \varphi_{i}(\theta)
$$

Herein, the same accuracy is used as for the pivoted Cholesky decomposition which yields nearly the same ranks $m$. Moreover, the random variables are i.i.d. and modeled as uniformly distributed in $[-2 / \sqrt{3}, 2 / \sqrt{3}]$. The numerical computation of the shape functional, shape gradient and shape Hessian is now performed by a fast boundary element method as outlined in [12]. The Monte-Carlo method uses 10000 samples. As seen in Figures 6-8, the asymptotic expansions fit well the behaviour of the expectation and variance of the stochastic shape functional under consideration for all covariance kernels under consideration.

\subsection{The first eigenvalue of the Dirichlet-Laplace operator in a perforated ellipse}

To consider another output function, we present simulations for the first eigenvalue of the Dirichlet-Laplace operator. Again, the related shape gradient and shape Hessian are obtained thanks to Lemma 2.2. Here, the reference domain $D$ is the ellipse of semi-axes 4 and 3 where the disk of radius 1 which is centered in the center of the ellipse has been removed. Random perturbations are applied to the disk's boundary. As in the first example, we take

$$
f(\theta)=\sum_{i=1}^{N} \alpha_{i}(\omega) f_{i}(\theta)
$$




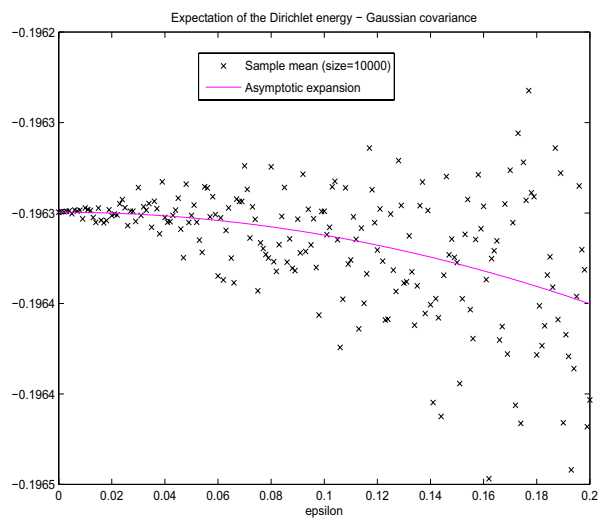

(a) Expectation

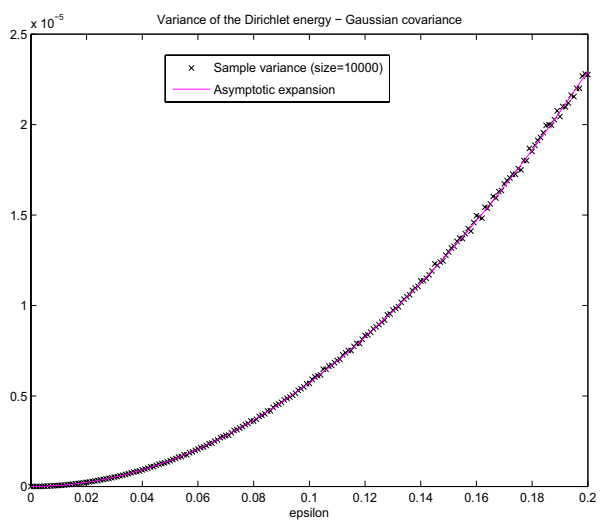

(b) Variance

Figure 6. Expectation and variance of the Dirichlet energy - Gaussian covariance kernel $k_{\infty}$.

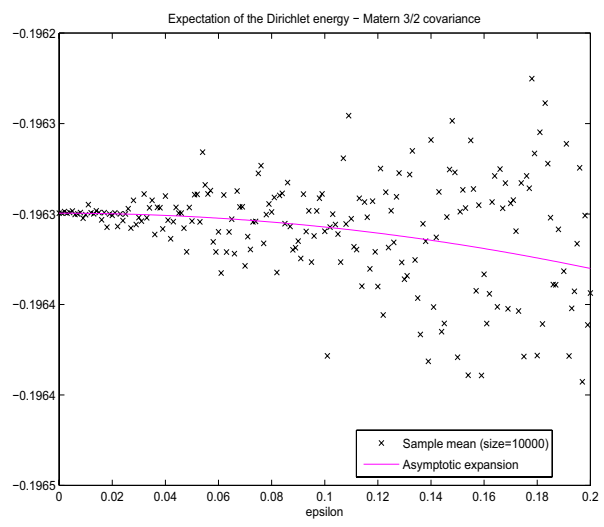

(a) Expectation

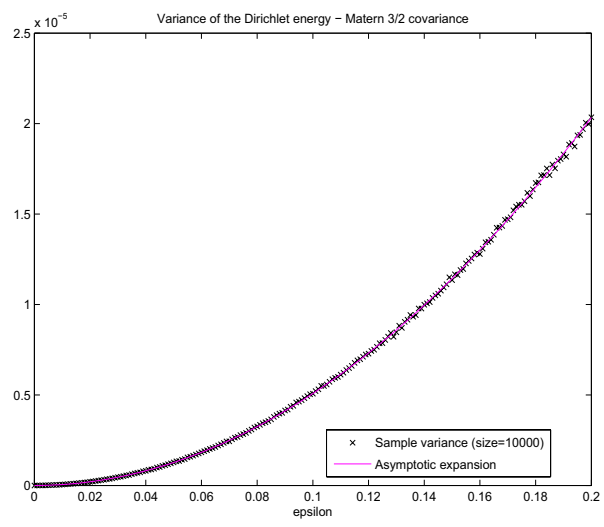

(b) Variance

FIgURE 7. Expectation and variance of the Dirichlet energy - Mátern covariance kernel $k_{3 / 2}$.

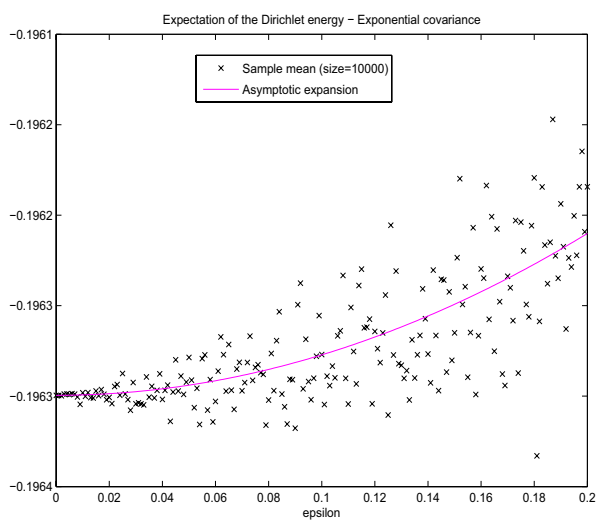

(a) Expectation

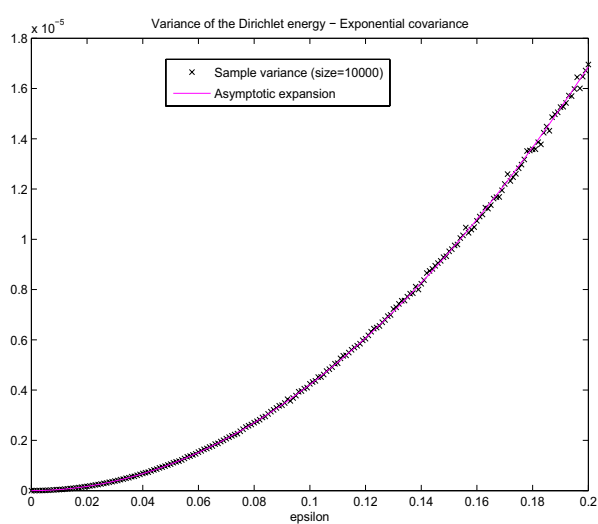

(b) Variance

FiguRE 8. Expectation and variance of the Dirichlet energy - Exponential covariance kernel $k_{1 / 2}$. 


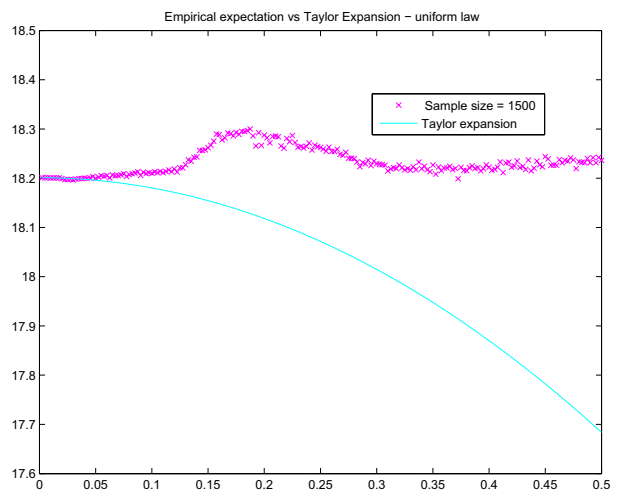

(a) Expectation

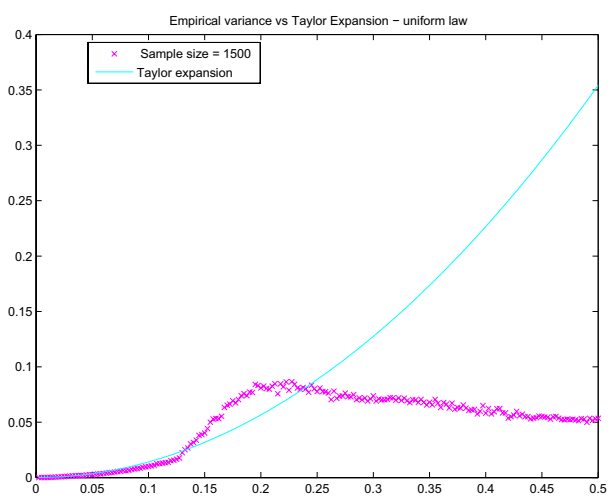

(b) Variance

Figure 9. Comparison between asymptotic expansion and Monte-Carlo simulation for the eigenvalue problem - Uniform distribution.

where the $\alpha_{i}$ are i.i.d. In the following computations, we have taken $N=21$ and the $f_{i}$ are the first normalized functions in the Fourier basis. We emphasize the local character of the approximation which is clearly seen in Figure 9.

Acknowledgements. The first author thanks support from the ANR through the grants OPTIFORM and ARAMIS.

\section{REFERENCES}

[1] L. Afraites, M. Dambrine and D. Kateb, On second order shape optimization methods for electrical impedance tomography. SIAM J. Control Optim. 47 (2008) 1556-1590.

[2] G. Allaire, F. Jouve and A.-M. Toader, Structural optimization using sensitivity analysis and a level-set method. J. Comput. Phys. 194 (2004) 363-393.

[3] F. Caubet, Instability of an inverse problem for the stationary Navier-Stokes equations. SIAM J. Control Optim. 51 (2013) 2949-2975.

[4] F. Caubet and M. Dambrine, Stability of critical shapes for the drag minimization problem in Stokes flow. J. Math. Pures Appl. 100 (2013) 327-346.

[5] A. Chernov and C. Schwab, First order $k$-th moment finite element analysis of nonlinear operator equations with stochastic data. Math. Comput. 82 (2013) 1859-1888.

[6] M. Dambrine, On variations of the shape Hessian and sufficient conditions for the stability of critical shapes. RACSAM, Rev. R. Acad. Cien. Serie A. Mat. 96 (2002) 95-121.

[7] M. Dambrine and D. Kateb, On the shape sensitivity of the first Dirichlet eigenvalue for two-phase problems. Appl. Math. Optim. 63 (2011) 45-74.

[8] M. Dambrine and J. Lamboley, Stability in shape optimization with second variation. Preprint HAL-01073089 (2014).

[9] M.C. Delfour and J.-P. Zolésio, Shapes and geometries. Metrics, analysis, differential calculus, and optimization. Vol. 22 of Advances in Design and Control, 2nd edition. Society for Industrial and Applied Mathematics (SIAM), Philadelphia, PA (2011).

[10] K. Eppler, Boundary integral representations of second derivatives in shape optimization. Discuss. Math. Differ. Incl. Control Optim. 20 (2000) 63-78.

[11] K. Eppler and H. Harbrecht, A regularized Newton method in electrical impedance tomography using shape Hessian information. Control Cybernet. 34 (2005) 203-225.

[12] K. Eppler and H. Harbrecht, Second-order shape optimization using wavelet BEM. Optim. Methods Softw. 21 (2006) 135-153.

[13] K. Eppler, S. Schmidt, V. Schulz and C. Ilic, Preconditioning the pressure tracking in fluid dynamics by shape Hessian information. J. Optim. Theory Appl. 141 (2009) 513-531.

[14] H. Harbrecht, A Newton method for Bernoulli's free boundary problem in three dimensions. Computing 82 (2008) 11-30.

[15] H. Harbrecht, On output functionals of boundary value problems on stochastic domains. Math. Methods Appl. Sci. 33 (2010) 91-102.

[16] A. Henrot and M. Pierre, Variation et optimisation de formes. Une analyse géométrique. Vol. 48 of Math. Appl. Springer, Berlin (2005). 
[17] H. Harbrecht, Second moment analysis for Robin boundary value problems on random domains, in Singular Phenomena and Scaling in Mathematical Models. Edited by M. Griebel. Springer, Berlin (2013) 361-382.

[18] H. Harbrecht, M. Peters and R. Schneider, On the low-rank approximation by the pivoted Cholesky decomposition. Appl. Numer. Math. 62 (2012) 428-440.

[19] H. Harbrecht, M. Peters and M. Siebenmorgen, Combination technique based $k$ th moment analysis of elliptic problems with random diffusion. J. Comput. Phys. 252 (2013) 128-141.

[20] H. Harbrecht, R. Schneider and C. Schwab, Sparse second moment analysis for elliptic problems in stochastic domains. Numer. Math. 109 (2008) 385-414.

[21] M. Hintermüller and W. Ring, An inexact Newton-CG-type active contour approach for the minimization of the Mumford-Shah functional. J. Math. Imaging Vision 20 (2004) 19-42.

[22] A. Novruzi and M. Pierre, Structure of shape derivatives. J. Evol. Equ. 2 (2002) 365-382.

[23] A. Novruzi and J.R. Roche, Newton's method in shape optimisation: a three-dimensional case. BIT (2000) 102-120.

[24] O. Pironneau, Optimal Shape Design for Elliptic Systems. Springer, New York (1983).

[25] S. Schmidt and V. Schulz, Impulse response approximations of discrete shape Hessians with application in CFD. SIAM J. Control Optim. 48 (2009) 2562-2580.

[26] V. Schulz, A Riemannian view on shape optimization. Found. Comput. Math. 14 (2014) 483-501.

[27] C. Schwab and R.A. Todor, Karhunen-Loéve approximation of random fields by generalized fast multipole methods. J. Comput. Phys. 217 (2006) 100-122.

[28] J. Simon, Second variations for domain optimization problems. In Control and estimation of distributed parameter systems. Edited by F. Kappel et al., Vol. 91 of Int. Ser. Numer. Math. Birkhäuser, Basel (1989) 361-378.

[29] J. Sokolowski and J.-P. Zolesio, Introduction to Shape Optimization. Springer, Berlin (1992).

[30] J. Wloka, Partial Differential Equations. Cambridge University Press, Cambridge (1987).

[31] S. Yang, G. Stadler, R. Moser and Omar Ghattas, A shape Hessian-based boundary roughness analysis of Navier-Stokes flow. SIAM J. Appl. Math. 71 333-355. 